On the basis of results obtained with dwarf mutants we attempted to treat sterile Ribes species hybrids with gibberellic acid and thereby to induce parthenocarpic fruit development. Flowers of Ribes culwerwelli Macfarl. [Ribes nigrun L. $X$ Grossularia reclinata f. uva crispa (L.) Janz.] and Ribes gordonianum Lem. (Ribes aureum Pursh. $\times$ Ribes sanguineum Pursh.) were sprayed with 50 p.p.m. gibberellic acid at the stage of full blossoming.

The above mentioned species hybrids were entirely sterile. In Ribes culwerwelli the formation of univalents ${ }^{3}$ ) and amphiplast $y^{4}$ ) was observed. Normal fruit development was only obtained in amphidiploids induced by colchicin treatment ${ }^{5}$ ) A few parthenocarpic fruits were obtained in the original diploid species in the course of unsuccessful back crossings ${ }^{6}$ ). Disturbances in cell division were found in both sexes of Ribes gordonianum $\left.\left.{ }^{7}\right),{ }^{3}\right)$. As the polyploidization of this species proved unsuccessful ${ }^{8}$ ) fruits were not obtained in spite of the heavy flowering of the plants ${ }^{8}$ ).

The treatment with gibberellic acid of the flowers of the above species proved successful. $87 \%$ parthenocarpic fruit setting was observed with Ribes culwerwelli and $31 \%$ with Ribes gordonianum. No parthenocarpic fruit development was observed in the control

The results obtained are particularly interesting from a theoretical point of view as new data have been provided for the overcoming of genetically blocked physiological deficiencies by physiological treatments. On the other hand it might be of interest to have a possibily for the morphological and chemotaxonomical comparison of the fruits of the interspecific hybrids (mainly Ribes gordonianum) with those of the parent varieties. Finally, the importance of practical application of gibberellic acid in the treatment of sterile hybrids should be stressed. The sterile hybrids were formerly regarded as useless. By the use of gibberellic acid these hybrid, if the quality of their fruits is suitable, can successfully be used for the production of seedless fruits.

Institute of Plant Breeding, Fertod, Hungary

J.M. ZATYKó

Eingegangen am 8. Oktober 1962

1) Brian, P.W., and H.G. Hemming: Physiol. Plantarum 8 669 (1955). - 2) Phinney, B. O.: Proc. Nat. Acad. Sci. U.S.A. 42, 185 (1956). - ${ }^{\text {a) }}$ MeUrman, O.: Hereditas 11, 289 (1928). - 4) KeEP, E.: Nature [London] $188,339(1060)-8)$ NiLsson, F.: Rep. XIV. Int. Hort. Cong. Netherlands, 1955, p. 697. - 6) Schmidr, M. Dtsch. Baumsch. 10, 280 (1952). - 7) Tischler, G.: Jb. wiss. Bot 42, 545 (1906). - 8) Nilsson, F.: Botan. Notiser 109, 33 (1956).

\section{Das „Entspannungsschwimmen“ von Velia und Stenus}

Nach einer kurzen Mittellung aus dem Jahre 1905') schwimmen Käfer der Gattung Stenus (Staphilinidae) unter bestimmten Bedingungen pfeilschnell über die Wasseroberfläche. Der Antrieb geschieht - wie wir in zahlreichen Versuchen bestätigen konnten - durch Abgabe eines entspannenden Sekretes aus einem Paar terminal stehender Abdominaldrüsen. Bei Vergleichsversuchen mit dem Bachwasserläufer Velia capraii konnte für diese Wanze ein analoger Mechanismus der Fortbewegung nachgewiesen werden. Bisher war von Velia nur Ruderschwimmen mit den Beinen bekannt ${ }^{2}$ ).

Markiert man die noch nicht entspannte Wasseroberfläche mit einer dünnen Schicht von Lycopodiumsporen, dann sieht man hinter Stenus wie Velia (Fig. 1) beim Entspannungsschwimmen" eine sich verbreiternde Bahn neugebildeter entspannter sporenfreier Oberfläche. Die gespannte, sich rasch zurückziehende Oberfläche reißt das auf ihr liegende kleine Insekt mit sich. Unter fortlaufender Sekretabgabe schwimmt so Stenus bipunctatus und St.biguttatus Strecken bis $15 \mathrm{~m}$; bei Velia jedoch beobachteten wir nur kurze Schwimmstöße von 10 bis $25 \mathrm{~cm}$ Länge. Die Geschwindigkeit von Stenus bipunctatus ist beim Entspannungsschwimmen mit 40 bis $75 \mathrm{~cm}$ / sec 25 bis 35 mal großer als beim normalen Schwimmen oder Laufen mit Hilfe der Beine. Der kurzen Strecken wegen konnten die entsprechenden Daten von Velia noch nicht ermittelt werden, doch treibt auch hier das Entspannungsschwimmen erheblich schneller voran als das Ruderschwimmen mit den Beinen.

Bei Velia hat von allen ausgequetschten Körperteilen nur der Inhalt der Speicheldrüsen (Maxillardrüsen ?) entspannende Wirkung, und nach Verkleben der Rüsselspitze war niemals mehr Entspannungsschwimmen nachzuweisen. Es ist daher zu folgern, daß beim Entspannungsschwimmen Speichel mit dem Rüssel nach hinten gespritzt wird.
Velia benutzt das Entspannungsschwimmen beim Start zur Flucht und, nach Freilandbeobachtungen, wahrscheinlich auch, um schnell eine rasche Strömung zu überwinden.

Bei Verwandten von Velia und Stenus, die nicht die Fähigkeit des Entspannungsschwimmens besitzen, wie z.B. der Staphilinide Philonthus varius und der Gerride Gervis lacustris,

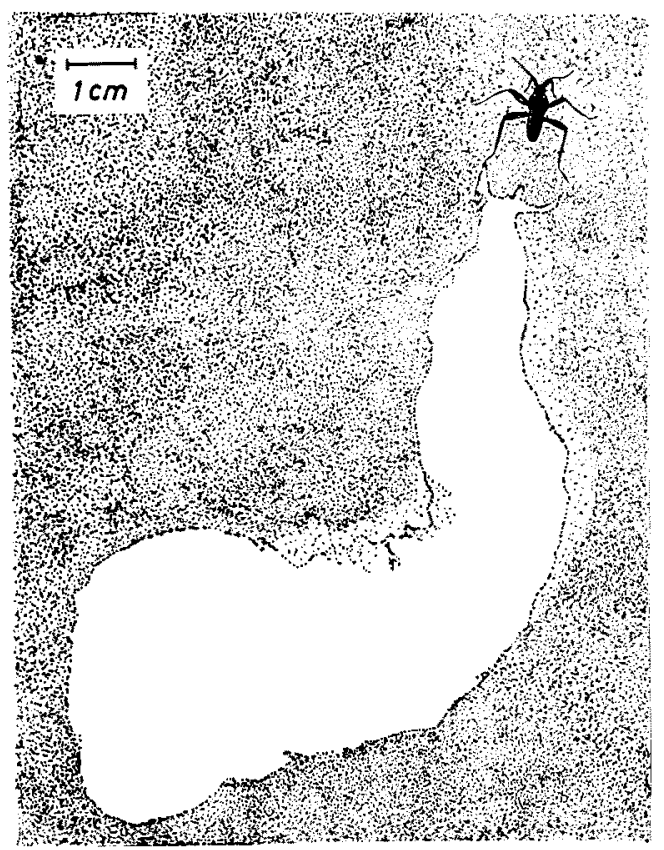

Fig. 1. Velia beim Entspannungsschwimmen. Entspannte Oberfläche weiB, gespannte punktiert. Nach einer Photographie

wirken die Sekrete der Abdominaldrüsen bzw. der Speicheldrüsen ebenfalls stark entspannend, so daß hier von einer Prädisposition zum Entspannungsschwimmen gesprochen werden kann. Die Abdominaldrüsen der Staphiliniden düften ihrer ursprünglichen Funktion nach Wehrdrüsen sein. Die Normal funktion der Speicheldrüsensekrete von Velia und Gerris dürfte in der Paralyse und Histolyse der Beutetiere bestehen.

Zoologisches Institut der Universität, Freiburg $i . \mathrm{Br}$.

\section{K. E. Linsenmair und $R$. Jander}

Eingegangen am 11. Dezember 1962

1) Billard, G., u. C. Bruyant: Compt. rend. hebd. soc. Biol. 2, 102 (1905). - 2) Rensing, L.: Zool. Beitr. 7, 3 (1962).

Zur Frage sog. jahreszyklischer Veränderungen an den Epithelkörperchen (Glandulae parathyreoideae) von Rana temporaria

Angeblich jahreszyklische Strukturveränderungen an den Epithelkörperchen (EK) von Anuren sind seit RoMEIS ${ }^{1}$ ) wiederholt festgestellt worden ${ }^{2}$ ), ${ }^{3}$ ), ${ }^{4}$ ). Um zu klären, ob sie durch Umweltfaktoren verursacht werden, hielten wir 80 Ranae temp. (R. t.) aus Herbstfängen über Winter bei 8 bis $10^{\circ} \mathrm{C}$. Sie blieben ohne Fütterung kräftig und laichten im März und April. - Von Januar bis April wurden ihre EK histologisch untersucht. Befunde: Die EK bestehen stets aus einem kompakten Epithelzellverband. Meist läßt sich eine zentrale Zone aus vorwiegend zytoplasmaarmen Zellen mit langgestreckten Kernen von einer peripheren Zone mit zytoplasmareicheren rundkernigen Elementen unterscheiden. - Somit entspricht die Struktur der EK jener, die man fast das ganze Jahr hindurch antrifft. Die für Februar oder März erwarteten jahreszyklischen Veränderungen waren ausgeblieben.

30 weitere R. t. aus Frühjahrsfängen wurden im Sommer bei 3 bis $5^{\circ} \mathrm{C}$ gehalten. Die Tiere verfielen in , Winterstarre" reagierten nur träge, wachten aber bei Frwärmung rasch auf. Die $E K$ von je $5 \mathrm{R}$. $t$. wurden nach einer bzw. vier Wochen entnommen. Ein Tier starb am 14. Versuchstag, vier weitere drohten zwischen dem 6. und 21. Tag einzugehen; ihre EK wurden ebenfalls untersucht. Die restlichen $15 \mathrm{R}$. t. wurden nach 4 wöchiger Versuchsdauer in einem Freigehege ausgesetzt; nach 3, 10 und 17 Tagen wurden auch ihre EK entnommen. An den EK von 10 R. t. waren eindeutige Strukturveränderungen eingetreten: vornehmlich in der peripheren Zone ist 